\title{
Expand distance education connotation by the construction of a general education cloud
}

\author{
Xu zhihong, Gu junhua, Dong yongfeng, Zhang jun, Li yan
}

Hebei university of technology, Tianjin, China

\begin{abstract}
:
With the development and popularization of cloud computing technology, education cloud appears gradually also. The construction of education cloud has the positive function for modern distance education to refrain from repetitive construction of $\mathrm{CAI}$ system and strengthen personalized teaching, remote experiment and so on. This paper designs a general education cloud computing platform suitable for the construction of teaching resources, teaching management, teaching service and innovative practice teaching, and discusses the key technology of safety management, service quality management, teaching resource management, and intelligent service management, etc.
\end{abstract}

Keyword: cloud computing; education cloud; distance education

\section{Introduction}

Cloud computing is based on data center technology, high-speed network technology and intelligent information processing technology, it deploys information processing tasks on a large number of distributed resource pool through the Internet, so that the computation ability, storage space, cyber source and information service has strong scalability. For information operators, it has obvious advantages in the aspects of hardware and software investment, application automatic deployment, and service quality improvement. For software developers, it has advantages of large scale virtual resource, platform versatility, high reliability, support reuse. For users, it can offer a variety of reliable online applications and services on demand cheaply at anywhere, at any time ,and in any way.

The construction of education cloud is conductive to solve the education problems of China, such as inequality in education and repetitive construction and waste of teaching resources, achieve social learning environment across the school and trans-regional, so everyone can access educational resources equally whenever and wherever. And, Chinese "Education informatization ten year development plan (2011-2020)" put forward the China Education Information cloud services platform construction task clearly.

Long-distance education has passed the paper-based media correspondence education, audio\&video based broadcast television teaching, and has entered into multimedia\&network based online distance education. The accumulation of teaching resources, teaching methods, and teaching management from all these development stages makes the construction of education cloud feasible.

\section{Design of a general education cloud architecture}

\subsection{Requirement analysis of general education cloud}

Education cloud should provide professional virtual software and hardware environment with varied services to schools, teachers, and students, which is cheap, easy to use and with high quality of service.

For educational institutions, education cloud should provide integrate management information system for network collaboration working. Support teaching resource storage, education application development, facilitate the development and expansion of education application service.

For teachers, education cloud should provide shared multimedia teaching resource library to store, manage, use and develop teaching resources.

For learners, it should provide a comprehensive learning and living environment with applications of education, instruction, entertainment, communication, and intelligent network learning; and for the related professional teachers and students, offer a virtual environment for professional innovative experiment and research.

\subsection{Architecture design of general education cloud}

According to the education cloud service requirement, general education cloud architecture is designed as Figure 1. It is consisted of six parts: user interface platform, Saas, Paas, Iaas, Physical platform, and service assurance platform.

Software as a service (Saas) platform provides comprehensive education application service according to teacher or student user's needs. Including collaborative working, teaching resource management, computer aided education and instruction, leisure life, billing and 
charging ,and user intelligent service management, etc.

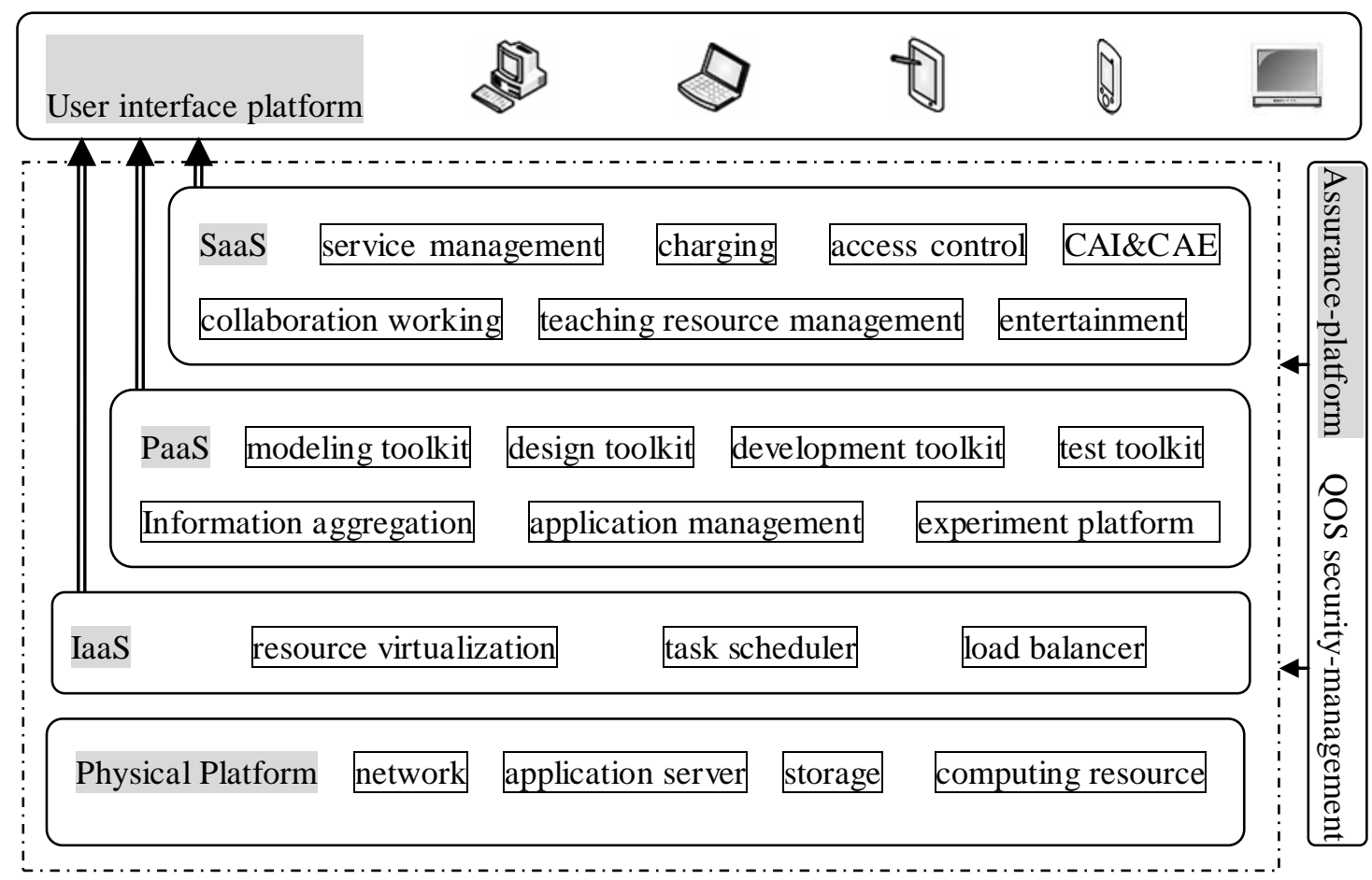

Figure 1: general education cloud architecture

Platform as a service (Paas) platform provides various tools for education application development and management. Modeling toolkits, application design toolkits, a variety of cloud computing application development toolkits, and testing toolkits can be used for education application development. Information aggregation middleware and application management middleware can be used for application component identification, authorization, heterogeneous data storage, and directory maintenance. Virtual experiment platform provide remote access interface of soft, hardware environment requirements for various innovative experiment hide the underlying.

Infrastructure as a service (Iaas) platform provide virtual experimental environment of distributed computing and data storage for teachers and students to do various distributed computing experiment. And implement resource management, task scheduling, and load balancing for physical resources, provide infrastructure for the upper layer.

Service guarantee platform provide quality of service management and security management service for each layer, to ensure the safety of the whole system, and achieve high quality service. User interface platform supports a variety of user terminal, to ensure consistency of access to a wide variety of terminal at any time, anywhere, to provide educational resource access service.

\subsection{Deployment model design of general education cloud}

The cloud deployment models can be classified into public cloud, private cloud, hybrid cloud, and community cloud. Public cloud computing service is open to users, it is the most convenient service mode, but the information security is difficult to ensure. Private cloud is established within the organization, information security is good, but the scope of services is limited. Hybrid cloud combines the characteristics of public cloud and private cloud, the key data are stored in private way in order to protect data privacy; and can guarantee the quality of service by public cloud resources in case of the private cloud overload. But, the seamless service migration of public cloud and private cloud is difficult. Community cloud integrate various resources within the region to provide public cloud computing services in the community.

For general education cloud, private cloud cannot satisfy the in-depth scientific research and outside service requirements, public cloud cannot conform to the safety requirements of coordinate working and teaching resources, so we need to design a compound cloud deployment architecture. Gradual evolution scheme is feasible: firstly, construct private cloud by schools for themselves, then form 
university community when some school's private cloud runs stable, and then transform to hybrid cloud or public cloud gradually when most related technology mature. Thus, with the development of cloud platform and function provision, general education cloud can not only supplement teaching management function and scientific practice environment to distance education, but also can integrate various of education resources to form more abundant distance education system.

\section{Key technologies}

Safety management, quality of service management, resource virtualization, huge data storage, intelligent information service are key problems of cloud computing, and are very important to general education Cloud.

\subsection{Safety management design}

In the cloud computing system, all information are stored in cloud server, the boundary of traditional network ceased to exist, the ownership and management of information are separated, information security prove to be acute issue. In general education cloud, comprehensive security strategies are designed to guarantee the security of the entire system:

(1) Use virtual resource management to isolate resources, to achieve security in the infrastructure layer effectively.

(2) Use firewall technology, real-time virus protection and intrusion detection to achieve real-time safety monitoring.

(3) Establish application development security system, adding safety management component in the application management middleware, to make safety record for all cloud application components.

(4) Create application threat model and detect application security, to prevent the cross-platform scripting (XSS) and SQL import in the application development layer.

(5) Through key data encryption store, digital signature and secure network protocol ensure the security of information transmission.

(6) In the service platform layer, establish classification user security group, use electronic visa to strengthen identity authentication, and adopt RBAC mechanism to strengthen access control.

\subsection{Service quality management design}

Cloud computing needs to provide not only the functional demand of resources and services for users, but also need to guarantee the quality of service (QoS), include availability, reliability, fault tolerance and time performance, etc. In general education cloud, comprehensive QoS strategies are designed to guarantee the quality of service:

(1) Use storage capacity, computing ability, communicate ability and application environment as direct basis for the virtual resource division.

(2) Set up resource allocation, monitoring, management function, to convenient the virtual resource service quality inspection and management in real time.

(3) Establish the QoS negotiation mechanism between layers, to guarantee the lower layer's service quality to the upper layer.

(4)Create dynamic, autonomous virtual resource pool of computing resources, storage resources, network bandwidth resources, and application service resource, etc., for dynamic allocation of resources according to the specific application of user's QoS requirements, to improve system availability and reliability.

(5) Through the rational use of virtual resources, and resources backup strategy, to improve the system fault tolerance.

(6)Use task scheduling and load balancing method based on real-time resource detection, to guarantee the performance of service time.

(7) Use distributed and redundant storage, to ensure the reliability and efficient of data storage, and to avoid "hot" data access, to improve the system throughput and transmission rate.

\subsection{Teaching resource management design}

Educational cloud system need to integrate all previous education information management system and CAI software, and add more management functions, such as all kinds of teaching resources management. It relates to different information forms, there are many structured data, as well as many semi-structured and unstructured information, involves massive data storage, multimedia information processing and information fusion technology:

(1)In the base layer of education cloud, design and build both structured database and unstructured database, and file system, to provide various forms of information storage service, achieve the deployment and configuration management of original information, middle information, and analysis result information in the distributed storage resources.

(2)Design information pipeline processing management components, to provide analysis, filtering functions and other custom and expansion policy to information flows, to ensure 
information availability, integrity, consistency, and security.

(3) Through the multi-level index policy, to implement the unified organization and management of multimedia teaching resources. Through information retrieval rights management and unified information access interface, to achieve safe and fast information retrieval.

(4) Design information channel component, support information queue, information publish, and information subscribe event driven, to provide concurrency information distribution capability.

(5) For multimedia information processing, use information fusion technology, to integrate teaching resources, to achieve multimedia courseware online making and sharing.

\subsection{Intelligent service management design}

Education cloud system aims at providing intelligent recommendation teaching service available at any time, at any where, and based on user demand. General education cloud architecture use knowledge component management, user behavior analysis, information aggregation and unified user interface, to provide intelligent cloud learning mode.

(1)Implementation teaching material's component management based on knowledge point, provide processing functions of knowledge material component's development, management, combination, and arrangement, support continual expanding of teaching resources, to facilitate the production of multimedia teaching must meet the functional requirements of the courseware teaching.

(2)Extract knowledge components from teaching resources, implement semantic analysis and relevance analysis of knowledge component, classify knowledge components by content aggregation, and then organize the knowledge components automatically.

(3)Establish behavior model of teachers, teaching manager, and cloud learners according to online statistical analysis of their network behavior history data log, and use intelligent fusion technology to achieve push type intelligent service to every kind of educational cloud users.

(4)Provide unified user interface, users can get applications on computer, mobile phone,
PDA, handheld computer or other telecommunication product at any time, at anywhere.

(5)Design comprehensive settlement functions, support multi-user terminal, multiple statistical certification, complete the information cloud learning service maintenance, management, billing, offline backup at the same time, provide a seamless extension of cloud learning support service.

\section{Summary}

With the development of cloud computing, cloud computing platform will support computer, TV, PDA, mobile phone and other terminal, the obtain of Cloud Applications and services will become more and more convenient, people can get education resources at any time and anywhere.

The general education cloud integrates teaching resources at all the levels of educational institutions, can effectively solve the problem of repeated construction of education resources. And through the development and application of intelligent Cloud technology, learners can achieve individualized teaching accordance with their aptitude. Use virtualization technology, can provide experimental environment to remote learners. All these play important role in extending the distance education connotation, constructing the lifelong education system, and establishing learning society.

\section{References}

[1] Zhang Jianxun, Gu Zhimin, Zheng Chao. Cloud computing research progress summary [J]. Application Research of computers,vol.27No.2 Feb.2010: 429-433

[2] Zhang Jinbao, Huang Ronghuai, Zhang liangang. Wisdom education cloud service: Research on the new model of education information service $[\mathrm{J}]$.Open education research, Vol.18No. 3 June.2012: 20-26

[3] Yang Xiaotang, Du Ruo, etc. The digital resources construction of the National Distance Education Based on cloud [J]. Chinese education informationization, Vol.21,2011: 14-17

[4] Yin Bingshan, Gao Qinli,etc. Open education courses resource pack [J]. Chinese distance education, April 2011: 65-69 